\title{
Effect of ${ }^{2} \mathrm{H}$ and ${ }^{18} \mathrm{O}$ water isotopes in kinesin-1 gliding assay
}

We show for the first time the effects of heavy-hydrogen water $\left({ }^{2} \mathrm{H}_{2} \mathrm{O}\right)$ and heavy-oxygen water $\left(\mathrm{H}_{2}{ }^{18} \mathrm{O}\right)$ on the gliding speed of microtubules on kinesin-1 coated surfaces. Increased fractions of isotopic waters used in the motility solution decreased the gliding speed of microtubules by a maximum of $21 \%$ for heavy-hydrogen and $5 \%$ for heavy-oxygen water. We also show that gliding microtubule speed returns to its original speed after being treated with heavy-hydrogen water. We discuss possible interpretations of these results and the importance for future studies of water effects on kinesin and microtubules. We also discuss the implication for using heavy waters in biomolecular devices incorporating molecular motors. 
1 Andy Maloney

2 College of Pharmacy

3 The University of Texas at Austin

4 Austin TX, USA

5 Lawrence J. Herskowitz

6 Fann Instrument Company

7 Houston TX, USA

8 Steven J. Koch

9 College of University Libraries \& Learning Sciences

10 The University of New Mexico

11 Albuquerque NM, USA

12 Corresponding author: Andy Maloney

13

14

15

16

17

18

College of Pharmacy

The University of Texas at Austin 2409 University Ave. Stop A1900

Austin, TX, USA 78712-1113

(512) 994-9502

amaloney@austin.utexas.edu 


\section{Introduction}

Water plays a crucial role in the interactions of biomolecules especially when biological surfaces bind and unbind. When binding surfaces are apart, water molecules form a hydration shell around the surfaces that is more ordered and less dynamic than bulk water molecules (Israelachvili and Wennerström, 1996). Changes in osmotic stress affect the rate of transport of water between the hydration shells around surfaces and the bulk water (Parsegian, Rand and Rau, 2000). Thus, onand off-rates for surface-surface binding is strongly affected by changes in the activity of bulk water. As noted by Parsegian, water activity changes that affect on- and off-rates are often overlooked, even in meticulous biophysical studies (Parsegian, Rand and Rau, 1995). In fact, water is an often-overlooked yet vitally important aspect for biophysical systems and is "the most important problem in science that hardly anyone wants to see solved" (Cho, Singh and Robinson, 1996). A good example of the profound importance of water activity is shown by a more than three orders of magnitude increase in binding lifetime in a protein-DNA complex when the osmotic stress of the system is increased by the addition of betaine (Sidorova and Rau, 2001). The effects of water activity have not been extensively studied in the molecular motors field, and in particular, for the kinesin-1 and microtubule system. In this study, we show the effects of changing the water isotope has on the speed of gliding microtubules using kinesin-1.

Though understudied for molecular motors, in general there have been some experiments indicating the importance of water on actin and myosin. For example, Highsmith et al. performed a detailed study on the effects of osmotic stress on the activity of myosin and binding to actin (Highsmith et al., 1996). They demonstrated that changing osmotic pressure was effective for probing water molecules at the binding interface. The authors also point out the importance of the increased osmotic pressure inside living cells. Chaen et al. also showed a $60 \%$ reduction in in vitro actin gliding velocity in heavy-hydrogen water (Chaen et al., 2001; Chaen et al., 2003).

We are unaware of prior studies involving use of water isotopes as osmotic stressors using the kinesin and microtubule system. However, there have been several studies of osmotic stress effects on tubulin alone and on microtubule polymerization. It is well known that osmotic stress promotes microtubule polymerization and a high percentage of glycerol is often used to promote in vitro microtubule polymerization (Shelanski, Gaskint and Cantor, 1973). It has also been shown that heavy-hydrogen water promotes tubulin polymerization and stabilization (Houston et al., 1974; Panda et al., 2000). Heavy-hydrogen water stabilizes polymerized microtubules, which has been shown to be a likely reason for why deuterium oxide is highly toxic to eukaryotic organisms (Kushner, Baker and Dunstall, 1999). Lewis initially demonstrated the toxicity of deuterium oxide in the 1930's (Lewis 1933; Lewis 1934). The ability of tubulin to polymerize fades rapidly when stored in normal aqueous buffer at $4^{\circ} \mathrm{C}$, however, heavy-hydrogen water dramatically reduces this instability allowing tubulin to polymerize even after storage at $4^{\circ} \mathrm{C}$ for 2 days (Chakrabarti et al., 1999). Heavy-hydrogen water has also been shown to stabilize a wide range of biomolecules and has potential technological implications for vaccine stability (Sen $e t$ $a l ., 2009)$. It is likely that heavy-hydrogen water has a general stabilizing effect on biomolecules and biomolecular complexes as it has also been shown to protect fruit flies from elevated temperature (Pittendrigh and Cosbey, 1974).

Despite its importance, it can be argued that water solvent effects have seen relatively little attention in the kinesin and microtubule system. Water isotopes have been used, but usually as spins or tracers rather than a probe of water activity (Hackney, Stempel and Boyer, 1980). In this paper, we show that heavy-hydrogen water and heavy-oxygen water each slow down microtubule gliding speeds on kinesin-1 surfaces. Thus, water isotopes may provide an important 
experimental knob to turn while studying the effects of water interactions on the behavior of kinesin- 1 in cells and biomolecular devices.

\section{Materials and Methods}

\section{Microscope}

Experiments were conducted on an Olympus IX71 inverted microscope using an Olympus 60x 1.42 NA PlanApo objective. Rhodamine fluorophores attached to tubulin were illuminated using a $100 \mathrm{~W}$ mercury lamp and excited using a TRITC filter cube from Chroma (Chroma 49005). The light illuminating the fluorophores was attenuated by $94 \%$ in order to prevent excessive photobleaching. The objective was temperature stabilized to achieve consistent microtubule speed measurements. A custom objective heater was constructed for the temperature stabilization and for a more in depth description of the heater please see (Maloney, Herskowitz and Koch, 2011). Briefly, the objective heater used a polyimide film resistive heater to heat the objective using control circuitry from TeTech (TC-48-20). LabVIEW software supplied by TeTech was used and modified to suit our time-stamping requirements. Temperature stabilization plays a crucial role in obtaining stable measurements (Highsmith 1977; Hackney, Stempel and Boyer, 1980; Böhm et al., 2000; Böhm, Stracke and Unger, 2000); however, many studies do not indicate the temperature at which speed measurements were observed. All our experiments were conducted at $33.1 \pm 0.1^{\circ} \mathrm{C}$. Image sequences were captured every $200 \mathrm{~ms}$ for a total of 600 frames -approximately 2 minutes — with an Andor Luca S EMCCD camera. Images were stored in "png" format using custom LabVIEW software.

Flow cells

Flow cells were constructed using two strips of double stick tape (Scotch), sandwiched between a microscope slide (VWR 48300-025) and a cover slip (VWR 48366-045). The channel formed between the pieces of tape was approximately $10 \mu \mathrm{L}$ in volume and was sealed using nail polish to prevented excessive evaporation of the motility solution during observations. In order to determine if heavy-hydrogen water affected speed measurements, we also created flow cells that could be "sealed" and "unsealed" with cellophane. A complete description of our procedure can be found in Supplemental text S1. Briefly, resealable flow cells were constructed in the same manner as sealable ones with the addition of two very thin pieces of double stick tape placed on top of the slip and at the entrances of the channel. Cellophane (Glad Cling Wrap) was then wrapped around the entrances of the flow cell in order to prevent the motility solution from evaporating.

\section{Buffers and solutions}

We used four different solvents to make the buffer solutions used in our experiments; light water $\left(\mathrm{H}_{2} \mathrm{O}\right)$, heavy-hydrogen water $\left({ }^{2} \mathrm{H}_{2} \mathrm{O}\right)$, heavy-oxygen water $\left(\mathrm{H}_{2}{ }^{18} \mathrm{O}\right)$, and deuterium-depleted light water.

The light water buffer was made at a 10x concentrated solution and contained: $800 \mathrm{mM}$ PIPES (Sigma 80635), $10 \mathrm{mM}$ EGTA (Sigma 80635), $10 \mathrm{mM} \mathrm{MgCl} 2$ (Sigma M1028) and pH-ed to 6.89 using approximately 1.25 M NaOH (Fisher S318). The light water used for this buffer had a resistivity of $18.2 \mathrm{M} \Omega-\mathrm{cm}$ and was produced with a Barnstead EasyPure RoDI system. The light water buffer was passed through a $0.2 \mu \mathrm{m}$ syringe filter and aliquoted in $2 \mathrm{~mL}$ screw-top vials, stored at $4^{\circ} \mathrm{C}$, and stored for no longer than 6 months. 
Another 10x concentrated buffer solution containing the same $800 \mathrm{mM}$ PIPES, $10 \mathrm{mM}$ EGTA, and $10 \mathrm{mM} \mathrm{MgCl}_{2}$ was prepared using heavy-hydrogen water (Sigma 151882) and pH-ed to a value of 7.30 using $\mathrm{NaOH}$. In order to report the correct $\mathrm{pD}$ value of a solution containing heavyhydrogen water, 0.41 must be added to the measured value of $\mathrm{pH}$ (Covington et al., 1968). Thus our solution had a $\mathrm{pD}$ value of 7.71, which is higher than the $\mathrm{pH}$ value for the light water buffer. The difference in the $\mathrm{pH}$ values of these two buffers and the consequence of those differences in the measured speed value is discussed below. The heavy-hydrogen water buffer was syringe filtered using a $0.2 \mu \mathrm{m}$ filter and aliquoted in $2 \mathrm{~mL}$ screw-top vials, stored at $4^{\circ} \mathrm{C}$, and stored for no longer than 6 months.

The third buffer solution contained heavy-oxygen water (Sigma 329878). A 90\% dilution of the 10x light water buffer was added to the heavy-oxygen water in order to create a solution containing $80 \mathrm{mM}$ PIPES, $1 \mathrm{mM}$ EGTA, and $1 \mathrm{mM} \mathrm{MgCl}_{2}$, which was the motility buffer conditions. We diluted the light water buffer into the heavy-oxygen water due to the high cost of the heavy-oxygen water. The solution was not filtered for fear of losing too much material and stored at $4^{\circ} \mathrm{C}$ for no longer than 6 months.

The fourth buffer again was a 10x concentrated solution and contained $800 \mathrm{mM}$ PIPES, $10 \mathrm{mM}$ EGTA, $10 \mathrm{mM}$ anhydrous $\mathrm{MgCl}_{2}$ (Sigma 449172) and $\mathrm{pH}$-ed to 6.89 with $\mathrm{NaOH}$ using deuterium-depleted light water as the solvent (Sigma 195294). The deuterium-depleted light water was quoted to have less than $1 \mathrm{ppm}$ deuterium oxide. The deuterium-depleted light water buffer was made with anhydrous $\mathrm{MgCl}_{2}$ in order to reduce the amount of contaminant deuterium in solution. It was syringe filtered using a $0.2 \mu \mathrm{m}$ filter and aliquoted into $2 \mathrm{~mL}$ screw-top vials, stored at $4^{\circ} \mathrm{C}$ for no longer than 6 months.

1x concentrated solutions of the light water buffer, heavy-hydrogen water buffer, and deuteriumdepleted light water buffer were made with the addition of $1 \mathrm{mg} / \mathrm{mL}$ bovine $\alpha$-casein (Sigma C6780) in solution in order to passivate the glass slide for the gliding motility assay. Bovine $\alpha$ casein was chosen as the surface passivator as it has been shown to give consistent gliding motility assays (Maloney, Herskowitz and Koch, 2011), however, it is not the only passivator that can be used to conduct gliding motility assays (Miller-Jaster, Petrie and Guilford, 2012). No $\alpha$ casein solution using the heavy-oxygen water buffer was made due to the cost of the $\mathrm{H}_{2}{ }^{18} \mathrm{O}$ water. The light water buffer containing $\alpha$-casein was used for the surface passivation when using heavy-oxygen water buffers. All $\alpha$-casein solutions were mixed for approximately one hour at room temperature using a stir plate until no visible precipitates of $\alpha$-casein were left in solution. These solutions were not filtered and were aliquoted into $2 \mathrm{~mL}$ screw-top vials and stored at $4^{\circ} \mathrm{C}$. No investigations were made to determine the solubility of $\alpha$-casein in the various water buffer solutions.

Tubulin was polymerized into microtubules using a Thermo PCR Sprint thermal cycler held at a constant temperature of $37^{\circ} \mathrm{C}$ for 30 minutes. The tubulin used for polymerization consisted of 29\% rhodamine-labeled bovine tubulin (Cytoskeleton TL331M) and 71\% unlabeled bovine tubulin (Cytoskeleton TL238) at a concentration of $5 \mathrm{mg} / \mathrm{mL}$. The polymerization solution was a total volume of $1 \mu \mathrm{L}$ and contained: light water buffer, an extra $1 \mathrm{mM} \mathrm{MgCl}, 1 \mathrm{mM} \mathrm{GTP}$ (Sigma G8877), 6\% (v/v) glycerol (EMD GX0185) and tubulin. Adding an extra $1 \mathrm{mM} \mathrm{MgCl}_{2}$ to the light water buffer ensured the EGTA did not chelate all the magnesium ions from solution since tubulin polymerization requires magnesium ions (Olmstedt and Borisy, 1975). Tubulin will polymerize in the presence of ATP; however, GTP is known to be a better nucleotide for polymerization (Desai and Mitchison, 1997). Glycerol acts as an osmotic stress in the polymerization solution and helps to speed up the polymerization process (Shelanski, Gaskint and 
Cantort, 1973). Microtubules were formed after 30 minutes in the thermal cycler and diluted by 200x with a solution of $10 \mu \mathrm{M}$ Taxol ${ }^{\mathrm{TM}}$ (Cytoskeleton TXD01) in light water buffer. Taxol ${ }^{\mathrm{TM}}$ is a non-polar chemical that must first be dissolved in DMSO (Sigma D2650) before adding to an aqueous solution. Taxol ${ }^{\mathrm{TM}}$ has been shown to create crystals in aqueous solutions due to its low solubility that appear to be fluorescent microtubules (Foss et al., 2008). It also has a high affinity for free tubulin and/or rhodamine dye molecules (Castro et al., 2009; Castro et al., 2010). In order to prevent Taxol ${ }^{\mathrm{TM}}$ crystals from forming in solution, all solutions requiring Taxol ${ }^{\mathrm{TM}}$ were prepared immediately before each experiment and no stock solutions containing Taxol ${ }^{\mathrm{TM}}$ in an aqueous environment were stored for more than the duration of daily experiments. Microtubules were left at room temperature for the duration of an experiment in order to prevent depolymerization (Shelanski, Gaskint and Cantort, 1973).

Dr. Haiqing Liu generously supplied Kinesin to us in $20 \mu \mathrm{L}$ aliquots at a concentration of 0.275 $\mathrm{mg} / \mathrm{mL}$ kinesin. The kinesin was his-tagged, truncated kinesin-1 dmk401 (Berliner et al., 1995; Asbury, Fehr and Block, 2003) from drosophila and was expressed in E. coli. Kinesin was diluted to $27.5 \mu \mathrm{g} / \mathrm{mL}$ for each assay.

\section{Motility assays}

All motility assays contained the following components; $10 \mu \mathrm{M}$ Taxol ${ }^{\mathrm{TM}}, 1 \mathrm{mM}$ Mg-ATP (Sigma A9187), 20 mM D-glucose (Sigma 49139), 2.5\% (v/v) of an oxygen scavenging antifade cocktail, and $5 \mathrm{~mL}$ of fixed polymerized microtubules. The antifade cocktail was a dual enzymatic oxygen scavenging system, which consisted of; 800 mg/mL glucose oxidase (Sigma G6641), 2000 $\mathrm{mg} / \mathrm{mL}$ catalase (Sigma C9322) and 20\% (v/v) of 2-mercaptoethanol, BME, (Sigma 63689). When diluted into the motility solution, there was $8 \mathrm{mg} / \mathrm{mL}$ glucose oxidase, $20 \mathrm{mg} / \mathrm{mL}$ catalase and $0.5 \%(\mathrm{v} / \mathrm{v})$ BME. Antifade cocktails were prepared in advance and stored in $5 \mathrm{~mL}$ aliquots at $-20^{\circ} \mathrm{C}$ and used within one week.

Experiments using light water required the flow cell to be passivated with $1 \mathrm{mg} / \mathrm{mL} \alpha$-casein in the $1 \mathrm{x}$ light water buffer for 10 minutes. Similarly, experiments using heavy-hydrogen water were incubated with $1 \mathrm{mg} / \mathrm{mL} \alpha$-casein in the $1 \mathrm{x}$ heavy-hydrogen water buffer, and those experiments using deuterium-depleted light water used $1 \mathrm{mg} / \mathrm{mL} \alpha$-casein in the $1 \mathrm{x}$ deuterium-depleted light water buffer. $1 \mathrm{mg} / \mathrm{mL} \alpha$-casein in the light water buffer was used as the passivator in the heavyoxygen water buffer experiments due to cost.

Each experiment incubated the flow cells at room temperature, $24^{\circ} \mathrm{C}$, for 10 minutes. During this 10 -minute incubation, kinesin was diluted to $27.5 \mu \mathrm{g} / \mathrm{mL}$ in a $20 \mu \mathrm{L}$ solution of either $1 \mathrm{x}$ light water buffer, 1x heavy-hydrogen water buffer, heavy-oxygen water buffer, or 1x deuteriumdepleted light water with the addition of $1 \mathrm{mM} \mathrm{Mg-ATP}$ and $0.5 \mathrm{mg} / \mathrm{mL} \alpha$-casein. After the 10 minute incubation with $\alpha$-casein, the $20 \mu \mathrm{L}$ kinesin solution was introduced to the flow cell by fluid exchange. The flow cell was then allowed to incubate for another 5 minutes. During this second incubation time, a motility solution was prepared using the different water isotope buffers. After the 5-minute incubation, $20 \mu \mathrm{L}$ of the motility solution was flown into the flow cell by fluid exchange. The flow cell was then sealed and observations were conducted immediately on the microscope. All observations were done in the center of the flow cell far from the tape and nail polish boundaries since this region gave the most consistent gliding motility speeds (Maloney, Herskowitz and Koch, 2011).

\section{Experiment and data collection}


Images were captured every $200 \mathrm{~ms}$. A total of 600 frames-approximately 2 minutes-were captured for a given region of interest (ROI) using an EMCCD gain of 150 with an Andor Luca $S$ camera. The use of the antifade cocktail, attenuation of the mercury lamp, and proper Köhler illumination helped prevent excessive photobleaching of the sample. A total of 15 ROIs were taken for each slide prepared. Due to the incubation of the flow cells at room temperature, it took approximately five 2 minute intervals until the slide reached a stable temperature set by the objective heater from which consistent speed measurements were achieved. Each water condition was repeated for a total of 3 independent flow cells with 15 ROIs taken for each sample.

Images were analyzed using custom LabVIEW 7.1 software to track and report the speed of each microtubule in an ROI. NI Vision 7.1 image segmentation algorithms via pattern matching identified microtubules. Tracking of microtubules was stopped if the microtubule end was within a few pixels from the boarder of the ROI or if a microtubule overlapped with another microtubule. If a microtubule had fewer than 100 consecutive image frames without tracking problems, the track was discarded. Microtubules that had a segmented area less than 55 pixels were also discarded. These values were determined empirically and were found to provide welltracked microtubules with few tracking errors.

Tracking provided the $\mathrm{x}$ and $\mathrm{y}$ position of microtubule ends with sub-pixel accuracy. This time series data was smoothed using a Gaussian window with a standard deviation of 2 seconds in order to eliminate transverse Brownian noise. Figure 1 shows a typical position trace with speed data from a microtubule using the light water buffer. The markers in Figure 1A are of the raw speed data obtained from the raw position data in 1B, also shown as markers. The smoothed position data in 1B (solid magenta line) is then used to calculate the smoothed speed data of the microtubule in 1A (solid magenta line). Smoothed data within 5 seconds of the beginning and end of a microtubule track were discarded to eliminate edge effects due to the Gaussian smoothing and are shown as the black solid lines in Figure 1A. The solid magenta line in 1A was the data used to calculate speed values for microtubules.

Speed versus time data for all the microtubules in an individual ROI were then concatenated together and the most likely speed was extracted using a kernel density estimation (KDE) using a Gaussian kernel of width $50 \mathrm{~nm} / \mathrm{s}$ (Silverman 1986). Using a KDE method instead of determining a simple mean speed reduced our sensitivity to microtubule pausing, stalling, or tracking errors. Figure 2 shows smoothed speed variations when using different water isotopes in the motility solution. 2A shows truncated smoothed speed data concatenated together for the heavy-hydrogen water buffer. It is clear that the microtubules do not travel at a single speed and can go through periodic pausing or stalling events. As greater amounts of the different water isotopes were added to the motility solution, microtubules began slowing and more of them had more instances of stalling or pausing. Figure 2B shows concatenated sorted smoothed speed values for the heavyhydrogen and heavy-oxygen water buffers as compared to a light water buffer (solid black line). Sorting the speed values clearly shows the effects of the water isotopes on microtubule speeds, as there are a greater number of lower speed values as compared to the light water buffer.

Figure 3 shows the results of a light water buffer assay where a simple histogram of smoothed concatenated speed values was generated using a $30 \mathrm{~nm} / \mathrm{s}$ bin width and the corresponding KDE analysis shown in the solid red line. Using the KDE analysis captures all the features of the histogram without the need to adjust bin sizes. It also allowed us to locate the most probable speed in which the microtubules within an assay were traveling at with a higher degree of accuracy. The large kernel width also reduced the sensitivity to speed changes due to the number of kinesin molecules pulling on a microtubule seen by (Gagliano et al., 2010). 


\section{Results}

Figure 4 shows speed variations using increasing amounts of heavy-hydrogen water (triangles) and heavy-oxygen water (circles) in the motility solution. Each data point represents three independent measurements where the error bars are the standard error of the mean. Several hundred microtubules were tracked for each data point at a constant temperature of $33.1 \pm 0.1^{\circ} \mathrm{C}$. The most likely speed using $100 \%$ light water was found to be $1016 \pm 11 \mathrm{~nm} / \mathrm{s}$ for the heavyhydrogen water experiment and $1007 \pm 4 \mathrm{~nm} / \mathrm{s}$ for the heavy-oxygen water experiment. Increasing the amount of either the heavy-hydrogen water buffer or the heavy-oxygen water buffer used in the motility solution caused the most likely speed measurement to decrease in a linear fashion. At the highest concentration of heavy-hydrogen water buffer used, the most likely gliding speed was found to be $799 \pm 8 \mathrm{~nm} / \mathrm{s}$ while with the heavy-oxygen water buffer was found to be $954 \pm 16 \mathrm{~nm} / \mathrm{s}$. The decrease in speed as compared to the light water buffer for the heavyhydrogen water was approximately $21 \%$ while for the heavy-oxygen water it was a decrease of $5 \%$. The deuterium-depleted light water buffer is also represented in this figure. The most probable speed at which the microtubules moved at in the deuterium-depleted light water buffer was found to be $1015 \pm 4 \mathrm{~nm} / \mathrm{s}$.

Figure 5 shows that heavy-hydrogen water does not cause permanent damage to the kinesin-1 surface. Using the resealable flow cell, we initially observed microtubules gliding in a light water motility solution for our standard 15 regions. We then exchanged the light water motility solution with twice the volume of the sample cell with the heavy-hydrogen water motility solution. We then resealed the flow cell with cellophane and observed microtubules in the heavy-hydrogen water buffer. As can be seen in Figure 5, the average measured speed of microtubules dropped from $987 \pm 2 \mathrm{~nm} / \mathrm{s}$ to $791 \pm 1 \mathrm{~nm} / \mathrm{s}$ when the light water motility solution was exchanged with the heavy-hydrogen water motility solution. In order to ensure that heavy-hydrogen water did not damage the kinesin-1 surface irreversibly, another fluid exchange from the heavy-hydrogen water motility solution back to the light water motility solution was done. Measured microtubule gliding speed values recovered back to $960 \pm 2 \mathrm{~nm} / \mathrm{s}$ thus indicating that heavy-hydrogen water does not damage the kinesin surface.

Comparing the methods of sealing the flow cell with cellophane and nail polish shows that using nail polish, as the sealant, does not interfere with motility. Nail polish contains organic solvents and other components that have the possibility to leach into the motility solution and cause damage to the assay. Measured speed values using both nail polish and cellophane as the sealant show that stable speeds can be measured and that nail polish does not damage motility observations over the time span of our experiments, 30 minutes for each slide.

\section{Discussion}

Adding light water buffer to the heavy-hydrogen water buffer to measure speed variations due to ${ }^{2} \mathrm{H}_{2} \mathrm{O}$ concentrations in the motility solution inevitably caused variations in the final $\mathrm{pH}$ of the solution. This was due to the fact that the light water buffer was $\mathrm{pH}$-ed to a value of 6.89 while the $\mathrm{pH}$ of the heavy-hydrogen water buffer was 7.30. In order for the two solutions to have the same effective $\mathrm{pH}$, the heavy-hydrogen water buffer should have been $\mathrm{pH}$-ed to 6.48, which would have made the $\mathrm{pD}$ 6.89. Böhm et al. showed that $\mathrm{pH}$ does affect the gliding speed of microtubules (Böhm et al., 2000). However, they showed that speed increases with increasing $\mathrm{pH}$ and showed that $\mathrm{pH}$ values between 6.89-7.71 increased measured gliding speeds by 
approximately $50 \mathrm{~nm} / \mathrm{s}$. Figure 4 shows that the speed difference from a solution containing all light water buffer ( $\mathrm{pH}$ of 6.89) and one containing nearly all heavy-hydrogen water buffer ( $\mathrm{pH}$ of 7.70) decreased measured speed values by approximately $200 \mathrm{~nm} / \mathrm{s}$. Due to the fact that our measured speed values are of greater magnitude and in the opposite direction expected from $\mathrm{pH}$ increase indicates that the effect we see is due primarily to isotope exchange and not to $\mathrm{pH}$ variation. Obtaining the $\mathrm{pH}$ value of 7.30 also required the addition of more $\mathrm{NaOH}$ to solution. Doing so also increased the ionic strength of the solution, which Böhm showed should increase the gliding speed, which is again opposite to the effect we observed (Böhm et al., 2000).

In light water, there exists approximately $17 \mathrm{mM}$ of deuterium in solution, which exist mostly as HOD molecules (Somlyai et al., 1993). In order to determine if this small amount of deuterium in solution affected the gliding speed of microtubules, a motility solution that was made of deuterium-depleted light water was used. Every possible effort was made to ensure that the trace amounts of deuterium in this motility solution were as low as possible. We found that the gliding speed of microtubules using a deuterium-depleted light water buffer was $1015 \pm 4 \mathrm{~nm} / \mathrm{s}$. This value agrees with the intersection of the fit line in Figure 4 and was indistinguishable from light water speed measurements. We were unable to detect effects of deuterium-depletion in the gliding motility assay, as expected.

There exists a measurable drop in speed values using the resealable flow cell between the initial observations using the light water motility solution and the final observations using the light water motility solution (see Figure 5). This drop in measured speed values was attributed to fluid exchanges in the resealable flow cell. Our initial experiments to determine the viability of a resealable flow cell used light water exclusively where we exchanged the fluid in the cell multiple times, see Supplemental text S2. Over successive fluid exchanges, the measured speed values decreased every time the fluid was exchanged and the resulting microtubule movement became untrackable after five fluid exchanges. The decrease in speed could be a result of kinesin and passivation being removed from the surface during successive fluid exchanges.

We observed that both heavy-hydrogen water $\left({ }^{2} \mathrm{H}_{2} \mathrm{O}\right)$ and heavy-oxygen water $\left(\mathrm{H}_{2}{ }^{18} \mathrm{O}\right)$ decrease the gliding speed of microtubules in a linear fashion. A similar decrease was observed for myosin and actin when substituting deuterium oxide for light water (Chaen et al., 2003). This indicates that both water isotopes affect a rate-limiting step for transport in the kinesin gliding assay. Kinesin bulk assays and single-motor assays suggest that the rate-limiting step is inorganic phosphate release and/or kinesin rear head release (Ma and Taylor, 1997; Moyer, Gilbert, and Johnson 1998; Guydosh and Block, 2009; Muthukrishnan et al., 2009). The rate-limiting step in the gliding assay has not been explored as thoroughly, and it may be affected by surface-effects not observed in bulk or single-motor assays. We note that the proportional decrease in gliding speed is similar to the proportional reported increase in viscosity of the two pure isotopic solutions (Hardy and Cottington, 1949; Kudish, Wolf and Steckel, 1972). We did not attempt to measure the viscosity of any of our fractional mixtures.

Increased microviscosity could be the cause of our observed decrease in gliding speed, which could possibly slow the rate of inorganic phosphate release (Blacklow, 1988; Benner, 1989). Microviscosity is also known as microscopic viscosity, which is the viscosity a single protein would be affected by due to the medium immediately surrounding it (Nemet, Shabtai, and Cronin-Golomb, 2002). If the effect we observed is due to microviscosity, then using water isotopes as a microviscosity probe could probe the effects of diffusion-controlled, rate-limiting steps in kinesin and other enzymes. Another method to perturb the viscosity of the system —and 
ultimately the osmotic pressure of the system-would be to use osmolytes such as betaine, sucrose, or polyethylene glycol. Changing the osmotic pressure of the gliding motility assay does affect microtubule gliding speeds and could also be used as a method for observing rate-limiting steps (Böhm et al., 2004). Thus, water isotopes, particularly $\mathrm{H}_{2}{ }^{18} \mathrm{O}$, which has less effect on hydrogen bonding than ${ }^{2} \mathrm{H}_{2} \mathrm{O}$, may provide important complementary information for osmotic stress studies.

Chaen et al. discuss the slowing of actomyosin in heavy-hydrogen water to be a result of a higher degree of hydrogen bonding to ADP within its motor domain thus indicating that the use of heavy-hydrogen water in the assay induces a slower hydrolysis of ATP (Chaen et al. 2001). Our results show a similar slowing effect of microtubules in the gliding motility assay due to an increase of heavy-hydrogen water and heavy-oxygen water used in the motility solution. The kinetic isotope effect on ATP hydrolysis in myosin using heavy-hydrogen water has been studied by several groups (Inoue, Fukushima and Tonomura, 1975; Inoue et al., 1975; Hotta and Morales, 1960), however, the kinetic isotope effect on ATP hydrolysis in heavy-oxygen water has not been studied. Further studies are required to indicate whether or not the slowing effect we observed is due to heavy-oxygen water affecting ATP hydrolysis or if our observations indicate effects due to microviscosity.

\section{Conclusions}

We have shown that microtubule gliding speeds on kinesin surfaces is systematically slowed by increasing the amount of heavy-hydrogen, ${ }^{2} \mathrm{H}$, or heavy-oxygen, ${ }^{18} \mathrm{O}$, water isotopes in the motility solution. The relative effects of heavy-hydrogen water versus heavy-oxygen water are similar to the relative viscosity changes seen in either water type for which microviscosity may provide an explanation for the effect, however, further experimentation and theoretical work is necessary to determine if heavy-oxygen water is causing a slowing of ATP hydrolysis. Water isotopes appear to be an effective experimental knob that can be used to study the effects of water on kinesin activity. Moreover, changing the water isotope (particularly the heavy-oxygen water substitution) could be an ideal method for perturbing the microviscosity of the solvent. Viscosity can be controlled to a certain degree by using different osmotic stress agents that affect viscosity differently. If indeed the effects from heavy-oxygen water are due to microviscosity, the information in this article can be used in combination with osmolyte studies to isolate the effects of osmotic stress.

Finally, some research groups are pursuing usage of molecular motor systems in microdevices (Bachand et al., 2004; Hess et al., 2005; Kim et al., 2013). It is feasible that heavy-hydrogen water is a superior solvent for these systems, due to its stabilizing properties, which include photostabilization (Sinha et al., 2002). We observed a 21\% speed decrease in microtubule gliding speeds when using $90 \%$ heavy-hydrogen water in the motility solution, which may be a demerit against the use of it in microdevices due to its effect on decreasing microtubule speeds. However, the time to catastrophe is greatly increased when microtubules are in heavy-hydrogen water and could outweigh the $21 \%$ decrease in speed due to the solvent if the microtubules were to last for several hours past when they would normally depolymerize. The use of molecular motors in microdevices adds a further need for understanding the behavior of kinesin and microtubules in differing water isotopes.

\section{References}


Asbury CL, Fehr AN, Block SM. 2003. Kinesin moves by an asymmetric hand-over-hand mechanism. Science 302: 2130-2134. doi:10.1126/science.1092985.

Bachand GD, Rivera SB, Boal AK, Gaudioso J, Liu J, Bunker BC. 2004. Assembly and transport of nanocrystal $\mathrm{CdSe}$ quantum dot nanocomposites using microtubules and kinesin motor proteins. Nano Letters 4: 817-821. doi:10.1021/n1049811h.

Benner SA. 1989. Enzyme kinetics and molecular evolution. Chemical Reviews 89: 789-806. doi:10.1021/cr00094a004.

Berliner E, Young EC, Anderson K, Mahtani HK, Gelles J. 1995. Failure of a single-headed kinesin to track parallel to microtubule protofilaments. Nature 373: 718-721. doi:10.1038/373718a0.

Blacklow SC, Raines RT, Lim WA, Zamore PD, Knowles JR. 1988. Triosephosphate isomerase catalysis is diffusion controlled. Biochemistry 27: 1158-1165. doi:10.1021/bi00404a013.

Böhm KJ, Stracke R, Baum M, Zieren M, Unger E. 2000. Effect of temperature on kinesin-driven microtubule gliding and kinesin ATPase activity. FEBS Letters 466: 59-62. doi:10.1016/S0014.-57939901757-3.

Böhm KJ, Stracke R, Unger E. 2000. Speeding up kinesin-driven microtubule gliding in vitro by variation of cofactor composition and physicochemical parameters. Cell Biology International 24: 335-341. doi:10.1006/cbir.1999.0515.

Böhm KJ, Stracke R, Vater W, Unger E. 2004. Inhibition of Kinesin-driven microtubule motility by polyhydroxy compounds. Hein H-J, Bischoff G, editors. In: Micro- and Nanostructures of Biological Systems. Aachen: Shaker Verlag GmbH, Germany. pp. 153-165. Available: http://lccn.loc.gov/2007440241.

Castro JS, Trzaskowski B, Deymier PA, Bucay J, Adamowicz L, Hoying JB. 2009. Binding affinity of fluorochromes and fluorescent proteins to Taxol crystals. Materials Science and Engineering: C 29: 1609-1615. doi:10.1016/j.msec.2008.12.026.

Castro JS, Deymier PA, Trzaskowski B, Bucay J. 2010. Heterogeneous and homogeneous nucleation of Taxol crystals in aqueous solutions and gels: effect of tubulin proteins. Colloids and Surfaces B: Biointerfaces 76: 199-206. doi:10.1016/j.colsurfb.2009.10.033.

Chaen S, Yamamoto N, Shirakawa I, Sugi H. 2001. Effect of deuterium oxide on actomyosin motility in vitro. Biochimica et Biophysica Acta 1506: 218-223. doi:10.1016/S00052728(01)00216-X.

Chaen S, Yamamoto N, Shirakawa I, Sugi H. 2003. In vitro actomyosin motility in deuterium oxide. Molecular and Cellular Aspects of Muscle Contraction 538: 183-192. doi:10.1007/978-1-4419-9029-7_17

Chakrabarti G, Kim S, Gupta ML, Barton JS, Himes RH. 1999. Stabilization of tubulin by deuterium oxide. Biochemistry 38: 3067-3072. doi:10.1021/bi982461r. 
Cho C, Singh S, Robinson GW. 1996. Liquid water and biological systems: the most important problem in science that hardly anyone wants to see solved. Faraday Discussions 103: 19-27. doi:10.1039/FD9960300019.

Covington AK, Paabo M, Robinson RA, Bates RG. 1968. Use of the glass electrode in deuterium oxide and the relation between the standardized $\mathrm{pD} . \mathrm{pa}_{\mathrm{D}}$ scale and the operational $\mathrm{pH}$ in heavy water. Analytical Chemistry 40: 700-706. doi:10.1021/ac60260a013.

Desai A, Mitchison TJ. 1997. Microtubule polymerization dynamics. Annual Review of Cell and Developmental Biology 13: 83-117. doi:10.1146/annurev.cellbio.13.1.83.

Foss M, Wilcox BWL, Alsop GB, Zhang D. 2008. Taxol crystals can masquerade as stabilized microtubules. PloS one 3: e1476. doi:10.1371/journal.pone.0001476.

Gagliano J, Walb M, Blaker B, Macosko JC, Holzwarth G. 2010. Kinesin velocity increases with the number of motors pulling against viscoelastic drag. European Biophysics Journal 39: 801-813. doi:10.1007/s00249-009-0560-8.

Guydosh NR, Block SM. 2009. Direct observation of the binding state of the kinesin head to the microtubule. Nature 461: 125-128. doi:10.1038/nature08259.

Hackney DD, Stempel KE, Boyer PD. 1980. Oxygen-18 probes of enzymic reactions of phosphate compounds. Methods in Enzymology 64: 60-83. doi:10.1016/S0076.68798064005-1.

Hardy RC, Cottington RL. 1949. Viscosity of deuterium oxide and water from $5^{\circ}$ to $125^{\circ} \mathrm{C}$. The Journal of Chemical Physics 17: 509-510. doi:10.1063/1.1747310.

Hess H, Clemmens J, Brunner C, Doot R, Luna S, Ernst K-H, Vogel V. 2005 Molecular selfassembly of "nanowires" and "nanospools" using active transport. Nano Letters 5: 629-633. doi:10.1021/n10478427.

Highsmith S. 1977. The effects of temperature and salts on myosin subfragment-1 and F-actin association. Archives of Biochemistry and Biophysics 180: 404-408. doi:10.1016/0003.-98617790054-6.

Highsmith S, Duignan K, Cooke R, Cohen J. 1996. Osmotic pressure probe of actin-myosin hydration changes during ATP hydrolysis. Biophysical Journal 70: 2830-2837. doi:10.1016/S0006.-34959679852-1.

Hotta K, Morales MF (1960) Myosin B nucleoside triphosphatase in deuterium oxide. Journal of Biological Chemistry 235: PC61-PC63.

Houston LL, Odell J, Lee YC, Himes RH. 1974. Solvent isotope effects on microtubule and depolymerization polymerization. Journal of Molecular Biology 87: 141-146. doi:10.1016/0022.-28367490566-X.

Inoue A, Fukushima Y, Tonomura Y (1975) Effects of deuterium oxide on elementary steps in the ATPase reactions evidence for the similarity of key intermediates in contractile and transport ATPase. The Journal of Biochemistry 78: 1113-1121. 
Israelachvili J, Wennerström H. 1996. Role of hydration and water structure in biological and colloidal interactions. Nature 379: 219-225. doi:10.1038/379219a0.

Kim E, Byun K-E, Choi DS, Lee DJ, Cho DH, Lee BY, Yang H, Heo J, Chung H-J, Seo S, and Hong S. 2013. Electrical control of kinesin-microtubule motility using a transparent functionalized-graphene substrate. Nanotechnology 24: 195102. doi:10.1088/09574484/24/19/195102.

Kudish AI, Wolf D, Steckel F. 1972. Physical properties of heavy-oxygen water. Journal of the Chemical Society, Faraday Transactions 1 68: 2041-2046. doi:10.1039/F19726802041.

Kushner DJ, Baker A, Dunstall TG. 1999. Pharmacological uses and perspectives of heavy water and deuterated compounds. Canadian Journal of Physiology and Pharmacology 77: 79-88. doi:10.1139/cjpp-77-2-79.

Lewis GN. 1933. The biochemistry of water containing hydrogen isotope. Journal of the American Chemical Society 55: 3503-3504. doi:10.1021/ja01335a509.

Lewis GN. 1934. The biology of heavy water. Science 79: 151-153. doi:10.1126/science.79.2042.151.

Ma Y-Z, Taylor EW. 1997. Interacting head mechanism of microtubule-kinesin ATPase. The Journal of Biological Chemistry 272: 724-730. doi:10.1074/jbc.272.2.724.

Maloney A, Herskowitz LJ, Koch SJ. 2011. Effects of surface passivation on gliding motility assays. PloS one 6: e19522. doi:10.1371/journal.pone.0019522.

Miller-Jaster KN, Petrie Aronin CE, Guilford WH. 2012. A quantitative comparison of blocking agents in the in vitro motility assay. Cellular and Molecular Bioengineering 5: 44-51. doi:10.1007/s12195-011-0202-y.

Moyer ML, Gilbert SP, Johnson KA. 1998. Pathway of ATP hydrolysis by monomeric and dimeric kinesin. Biochemistry 37: 800-813. doi:10.1021/bi9711184.

Muthukrishnan G, Zhang Y, Shastry S, Hancock WO. 2009. The processivity of kinesin-2 motors suggests diminished front-head gating. Current Biology 19: 442-447. doi:10.1016/j.cub.2009.01.058.

Nemet BA, Shabtai Y, Cronin-Golomb M. 2002. Imaging microscopic viscosity with confocal scanning optical tweezers. Optics Letters 27: 264-266. doi:10.1364/OL.27.000264.

Olmstedt JB, Borisy GG. 1975. Ionic and nucleotide requirements for microtubule polymerization in vitro? Biochemistry 14: 2996-3005. doi:10.1021/bi00684a032.

Panda D, Chakrabarti G, Hudson J, Pigg K, Miller HP, Wilson L, Himes RH. 2000. Suppression of microtubule dynamic instability and treadmilling by deuterium oxide. Biochemistry 39: 5075-5081. doi:10.1021/bi992217f.

Parsegian VA, Rand RP, Rau DC. 1995. Macromolecules and water: probing with osmotic stress. Methods in Enzymology 259: 43-94. doi:10.1016/0076.-68799559039-0. 
Parsegian VA, Rand RP, Rau DC. 2000. Osmotic stress, crowding, preferential hydration, and binding: a comparison of perspectives. Proceedings of the National Academy of Sciences of the United States of America 97: 3987-3992. doi:10.1073/pnas.97.8.3987.

Pittendrigh CS, Cosbey ES. 1974. On the very rapid enhancement by $\mathrm{D}_{2} \mathrm{O}$ of the temperaturetolerance of adult Drosophila. Proceedings of the National Academy of Sciences of the United States of America 71: 540-543.

Sen A, Balamurugan V, Rajak KK, Chakravarti S, Bhanuprakash V, Singh RK. 2009. Role of heavy water in biological sciences with an emphasis on thermostabilization of vaccines. Expert Review of Vaccines 8: 1587-1602. doi:10.1586/erv.09.105.

Shelanski ML, Gaskint F, Cantort CR. 1973. Microtubule assembly in the absence of added nucleotides. Proceedings of the National Academy of Sciences of the United States of America 70: 765-768. doi:10.1073/pnas.70.3.765.

Sidorova NY, Rau DC. 2001. Linkage of EcoRI dissociation from its specific DNA recognition site to water activity, salt concentration, and $\mathrm{pH}$ : separating their roles in specific and non-specific binding. Journal of Molecular Biology 310: 801-816. doi:10.1006/jmbi.2001.4781.

Silverman BW. 1986. Density Estimation for Statistics and Data Analysis. 1st ed. New York: Chapman and Hall.

Sinha S, Ray AK, Kundu S, Sasikumar S, Dasgupta K. 2002. Heavy-water-based solutions of rhodamine dyes: photophysical properties and laser operation. Applied Physics B 75: 85-90. doi:10.1007/s00340-002-0932-6.

Somlyai G, Jancsó G, Jákli G, Vass K, Barna B, Lakics V, Gaál T. 1993. Naturally occurring deuterium is essential for the normal growth rate of cells. FEBS Letters 317: 1-4. doi:10.1016/0014.-57939381479-J.

Yamada T, Shimizu H, Nakanishi M, Tsuboi M (1981) Environment of the tryptophan residues in a myosin head: A hydrogen-deuterium exchange study. Biochemistry 20: 1162-1168. doi:10.1021/bi00508a018. 


\section{Figure 1}

Characteristic tracking data from a microtubule in the light water buffer.

The markers in A) show the instantaneous speed of the raw tracking data for the trailing end of a single microtubule and the raw positional data is shown in B) as markers. The wide scatter of the markers in $A$ ) is due to transverse motion of the microtubule ends to the trajectory and obscures the gliding speed. To account for this, raw positional time series data were smoothed as described in the text, resulting in the solid magenta line in B). The solid black line in A) shows the instantaneous speed of the smoothed positional data. To account for edge-effects of smoothing, 5 seconds were removed from the beginning and end of the smoothed speed, resulting in the final speed dataset used for analysis, shown as the solid magenta line in A).

A

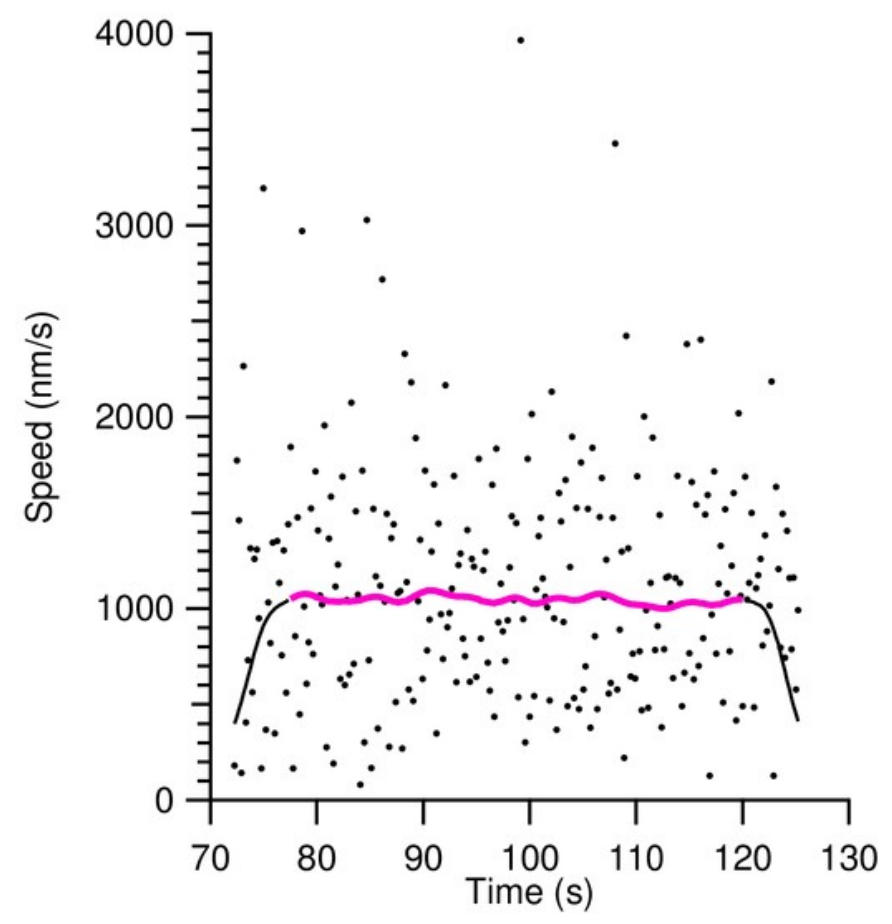

B

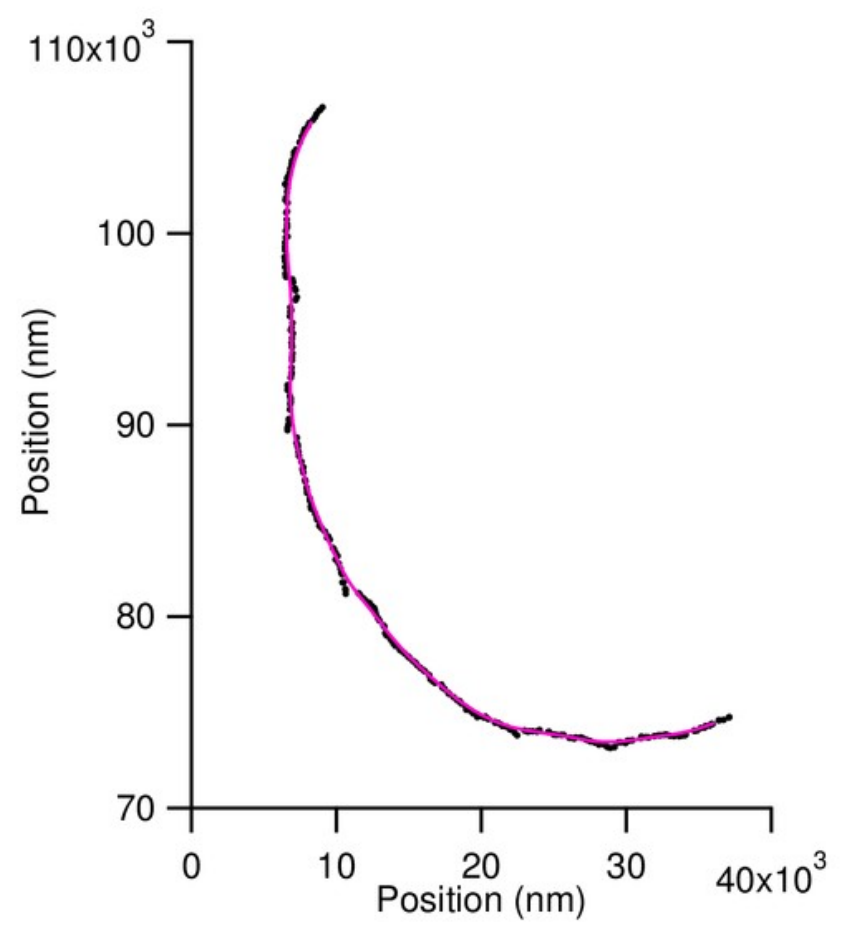




\section{Figure 2}

Concatenated smoothed speed values for varying amounts of water isotopes in the motility buffer.

A) Concatenated speed measurements for microtubules using heavy-hydrogen water. The black line has $0 \%$, blue $50 \%$, and red $90 \%$ heavy-hydrogen water in the motility solution. B) Sorting the speed values in A) shows the effects of pausing and stalling on microtubules in the various water isotopes. The solid lines used the heavy-hydrogen water and the dashed lines used the heavy-oxygen water. Solid lines have the same values as in A) and the green dashed line and purple dashed line had $45 \%$ and $81 \%$ heavy-oxygen water in the motility solution, respectively. 
A
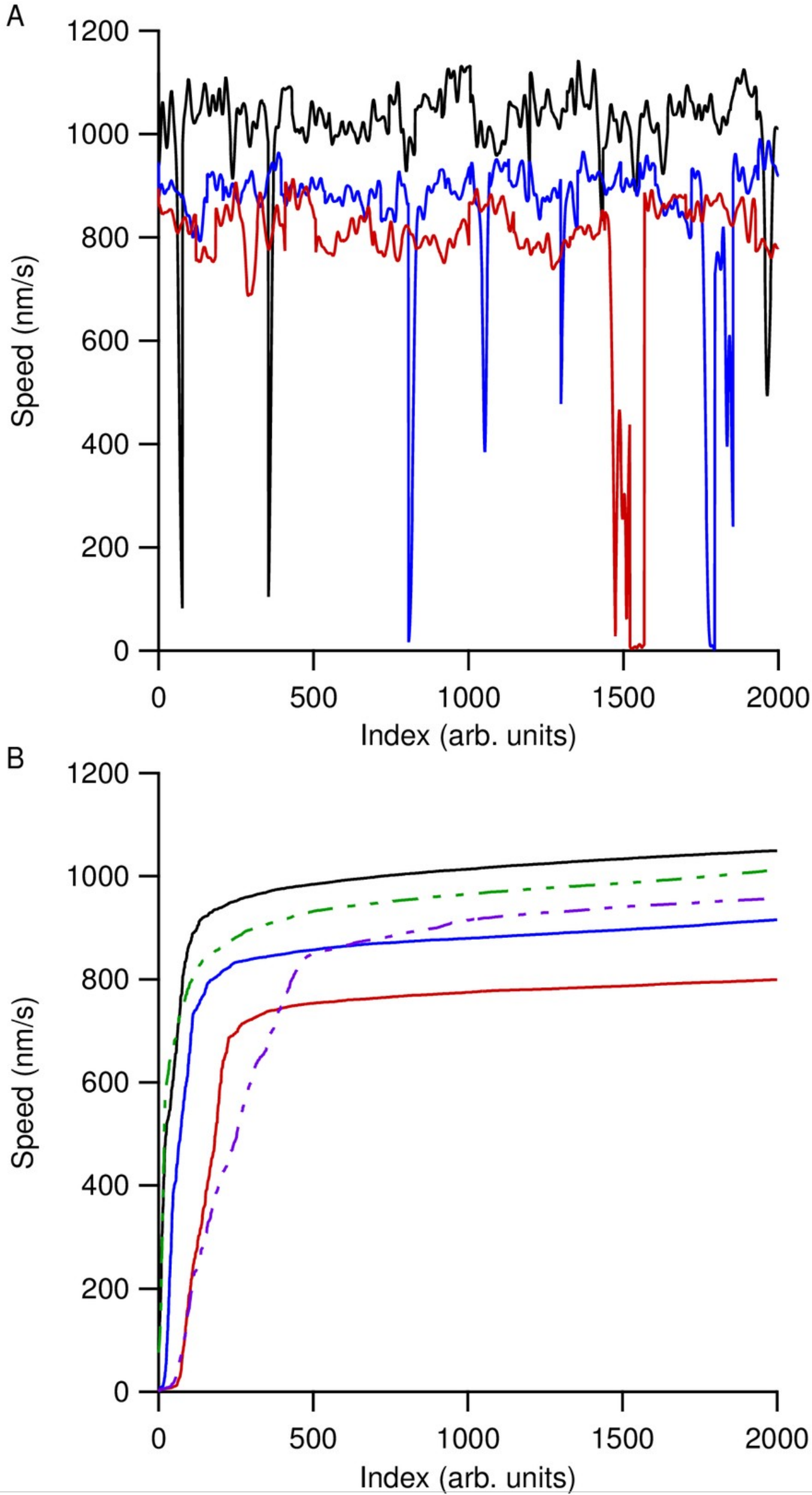


\section{Figure 3}

Comparison of the KDE algorithm with a histogram of smoothed concatenated speed values.

The bins correspond to a simple histogram of smoothed concatenated speed values using a $30 \mathrm{~nm} / \mathrm{s}$ bin size for a light water buffer assay. The figure corresponds to over 4000 speed points from over 400 different tracked microtubules. The red solid line is a KDE analysis of the smoothed speed values. The KDE algorithm correctly mimics the features of the histogram and allows for greater granularity when determining the most probable speed of microtubules within the assay.

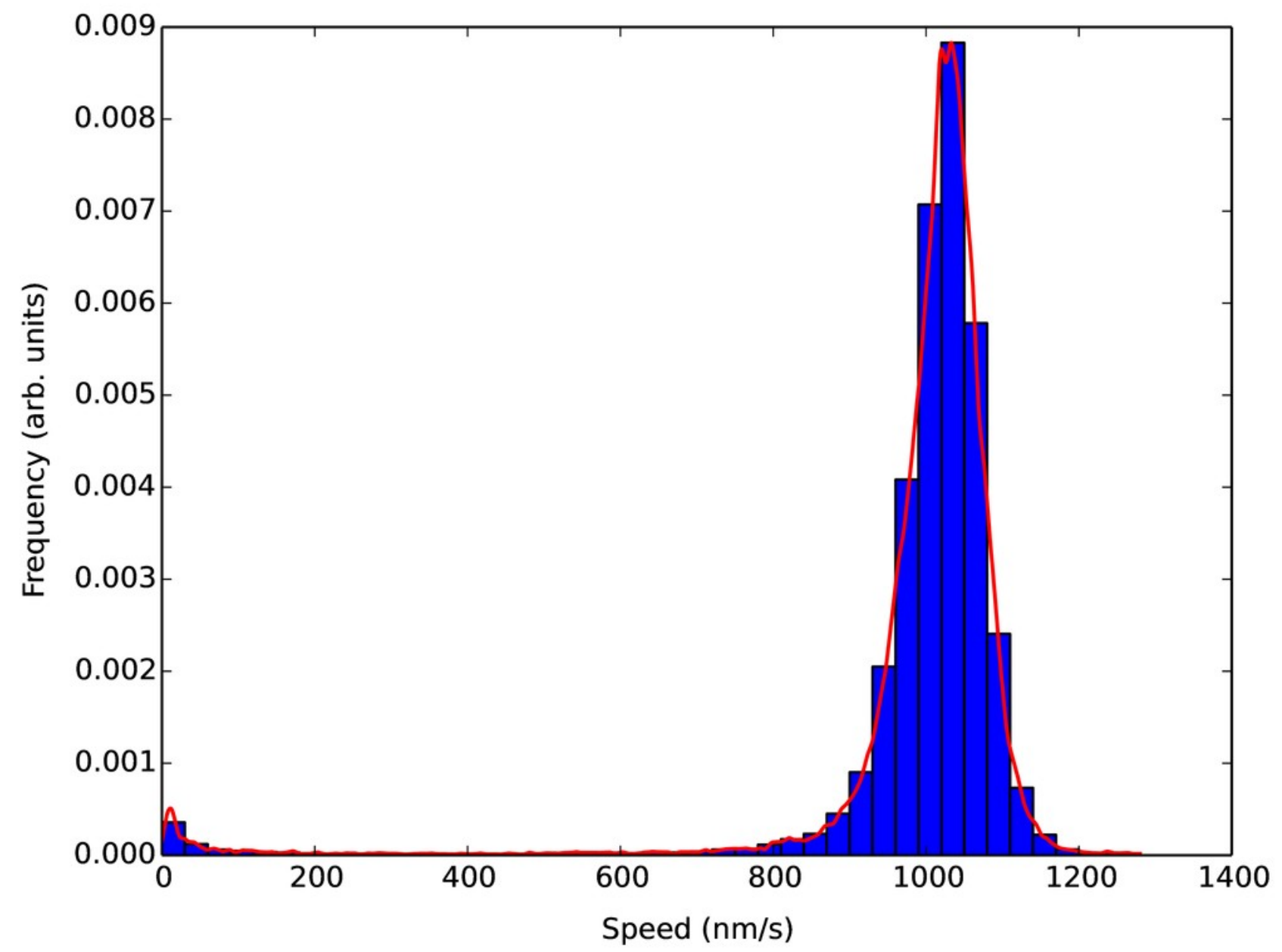




\section{Figure 4}

Microtubule gliding speed at $33.1 \pm 0.1^{\circ} \mathrm{C}$ versus concentration of water isotope in solution.

Markers represent the mean of observations from three independent samples. Triangles are the heavy-hydrogen water and circles are the heavy-oxygen water. Each of the three observations was an estimate of the most likely gliding speed of hundreds of microtubules in the sample after temperature equilibrium was reached (see text). Error Bars represent the standard error of the mean of the three observations. The solid line is a linear fit to guide the eye and does not represent a fit of a theoretical model. The steady decrease in speed (as opposed to a roll-off at intermediate concentration) indicates that the added deuterium affects a rate-limiting step for microtubule gliding speed on kinesin-1 surfaces. Not shown in the graph is the gliding speed in deuterium-depleted water (1015 $\pm 4 \mathrm{~nm} / \mathrm{s})$, which was indistinguishable from light water $(1016 \pm 11 \mathrm{~nm} / \mathrm{s})$. 


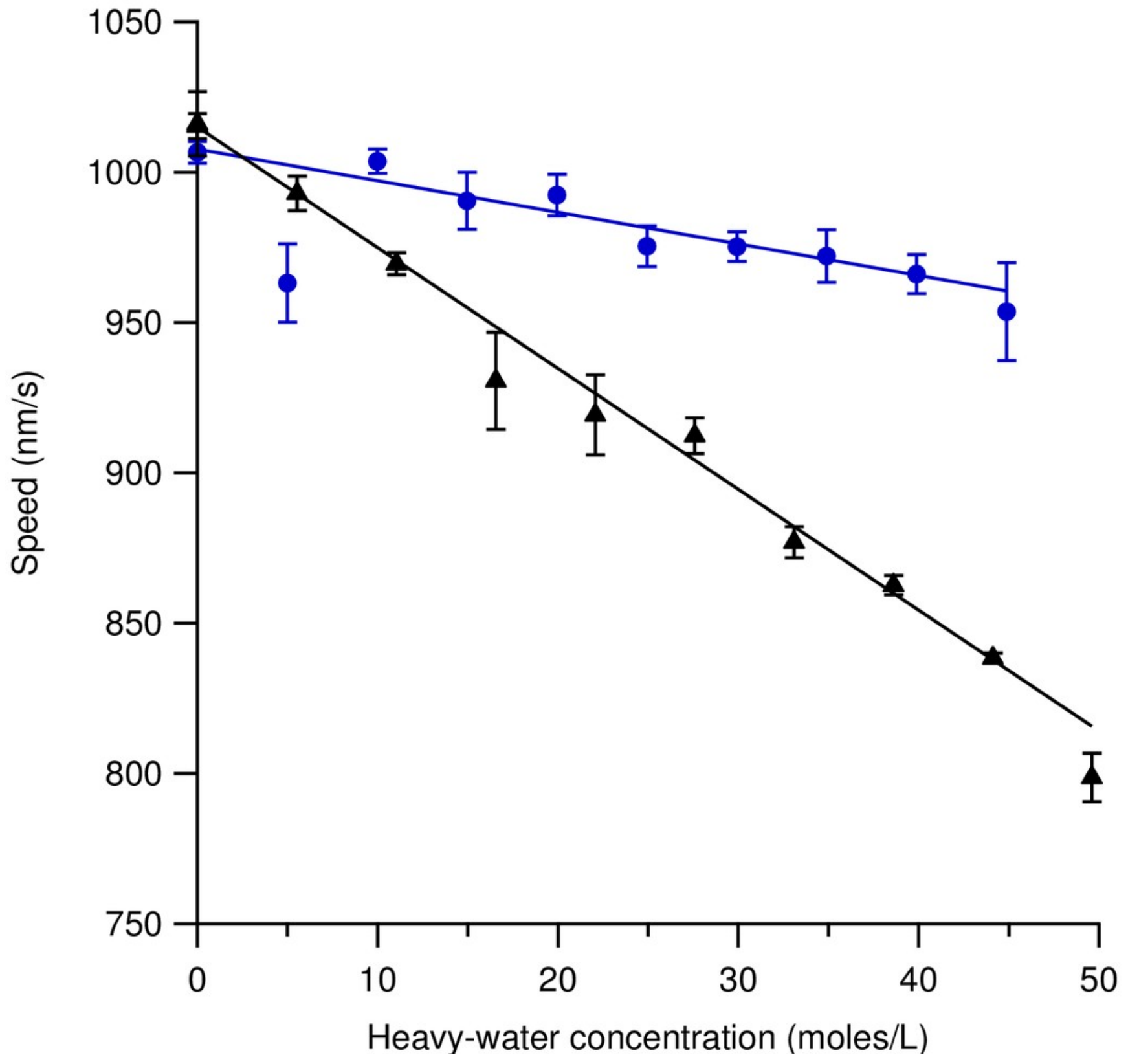




\section{Figure 5}

Determination of any deleterious effects of heavy-hydrogen water on the gliding motility assay.

Time is measured relative to the flow-in of the first motility buffer. Each solid circle represents the most likely gliding speed from the microtubules in one region of observation over a twominute period. Each set of measurements reveals the initial time necessary for the sample temperature to obtain equilibrium with the objective temperature. The first cluster of data represents observations with the light water buffer. An average speed of $987 \pm 2 \mathrm{~nm} / \mathrm{s}$ was measured for the last 10 data points. For all stated values, the first five data points were excluded due to the slide reaching an equilibrium temperature with the objective. The middle set of data represents observations after replacing the light water buffer with twice the volume of the flow cell with the heavy-hydrogen buffer. The measured average speed was $791 \pm 1 \mathrm{~nm} / \mathrm{s}$. The final set of data represents observations after re-filling the flow cell with twice the sample volume of light water buffer. The average measured speed was $960 \pm$ $2 \mathrm{~nm} / \mathrm{s}$. The return to nearly the same speed as the first data set indicates that heavy water does not cause severe permanent changes to the kinesin surfaces. Error bars are the standard deviation of the average for that ROI. 


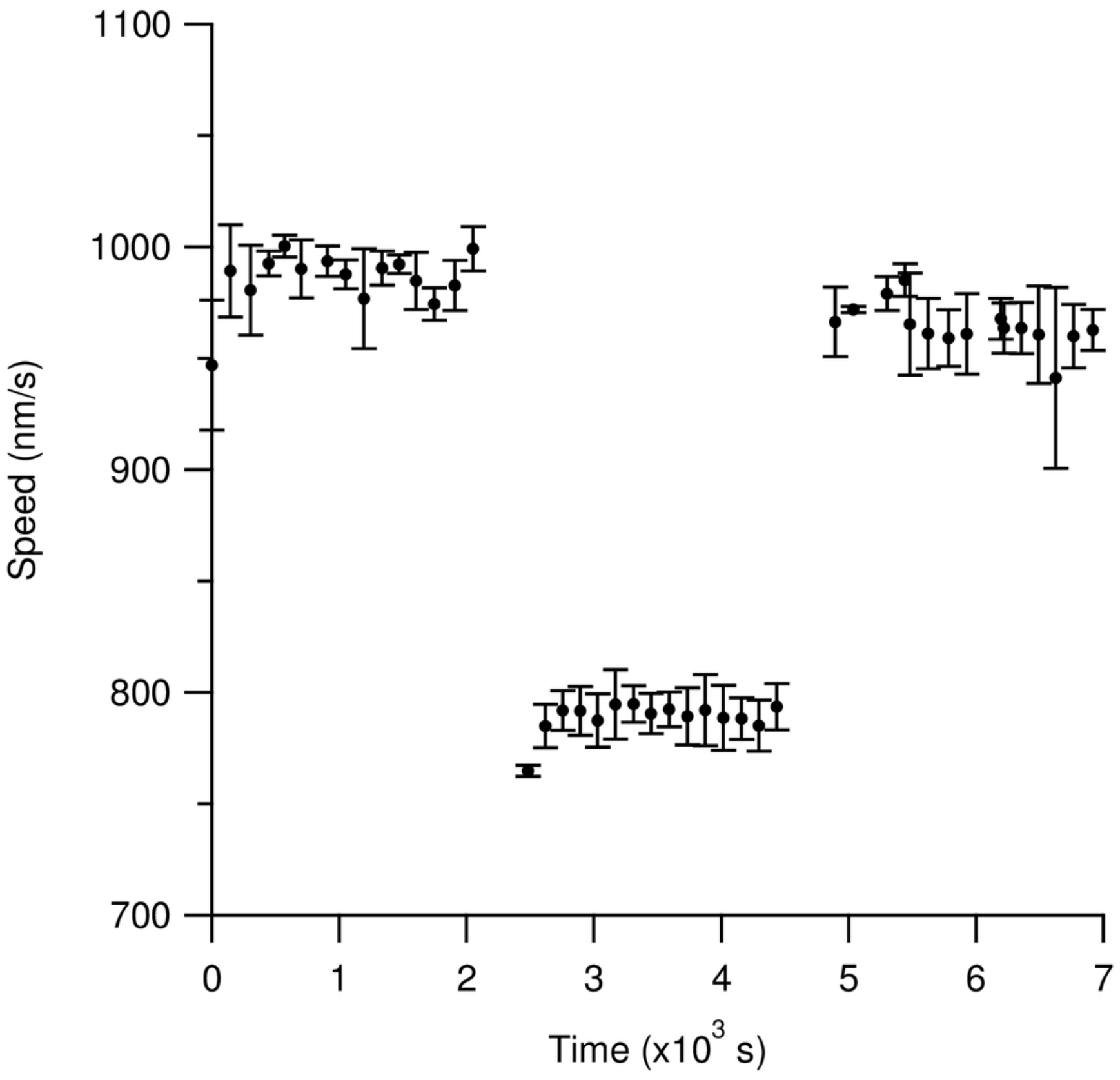

